\title{
IMPROVED STERNAL FIXATION IN THE TRANSSTERNAL BILATERAL THORACOTOMY INCISION
}

\author{
R. P. Brown, FRCS \\ D. S. Esmore, FRACS \\ C. Lawson
}

\begin{abstract}
The success of bilateral sequential single-lung transplantation has led to the rebirth of the transsternal bilateral thoracotomy incision. Despite the multiple advantages of this incision over a median sternotomy for this operation, one distinct disadvantage is the high prevalence of sternal disruption at the transverse sternotomy site, which is traditionally closed with sternal wires. This disruption rate is between $20 \%$ and $40 \%$ in most institutions and can lead to significant pain and deformity in addition to slowing the postoperative recovery and increasing the risk of sternal infection. To overcome this problem we have designed and manufactured a device that provides a sound method of fixating the sternum. The device is composed of two plates, one anterior and one posterior to the sternum, that are fixed to each other and to the sternum by means of screws into internally threaded posts positioned in predrilled holes through the bone on either side of the sternotomy. All components of the device are made from titanium and a range of post lengths are available to accommodate variability in sternal thickness. The structure of the device is demonstrated. The device has now been inserted into a total of 20 patients after bilateral lung transplantation and the preliminary results suggest this is a superior technique for closure of a transverse sternotomy and support continued and routine use. (J Thorac Cardiovasc Surg 1996;112:137-41)
\end{abstract}

$\mathrm{S}^{\mathrm{i}}$ ince the initial description of bilateral sequential single-lung transplantation in $1990,{ }^{1}$ this procedure has been rapidly establishing itself as the procedure of choice for end-stage septic lung disease and for end-stage emphysematous disease in a select group of patients.

The description of this procedure has led to the rebirth of the transsternal bilateral thoracotomy (or thoracosternotomy) incision, an incision once popular in cardiac operations before being abandoned for the simpler and less painful median sternotomy. This incision involves a bilateral submammary skin incision that is followed by transverse division of the pectoralis major muscles to allow access to the fourth intercostal spaces anteriorly. The intercostal muscles are then divided along with ligation and division of both internal thoracic artery pedicles.

From the National Heart and Lung Replacement Service/Baker Institute, Alfred Hospital, Melbourne, Australia.

Received for publication May 24, 1995; accepted for publication August 30, 1995.

Address for reprints: Robin P. Brown, FRCS, 4 Fraser St., Middle Park, Victoria, 3206, Australia.

Copyright (C) 1996 by Mosby-Year Book, Inc.

$0022-5223 / 96 \$ 5.00+0 \quad \mathbf{1 2 / 1 / 6 8 9 1 4}$
The pleura is then incised bilaterally, and to complete this incision the only remaining requirement is division of the sternum transversely at this level. The main advantage of this incision, also known as the clam-shell incision, is that it gives superb exposure to all aspects of both pleural cavities. This allows easy and safe division of adhesions, easy control of bleeding, and exposure to both lung hila. In addition, it allows rapid access to the heart if cardiopulmonary bypass is necessary. As familiarity with the incision increases, its use is expanding into other areas of cardiothoracic surgery particularly for bilateral pulmonary pathologic conditions.

One major disadvantage of this incision that is becoming prominent as the number of procedures increases is the high disruption rate at the transverse sternotomy site. This is traditionally closed with two or three sternal wires. The prevalence of this complication was $36 \%$ at our institution before introduction of our sternal fixation device and ranges from $20 \%$ to $60 \%$ at institutions worldwide. This disruption leads to increased and prolonged sternal pain in the initial postoperative period and increases the risk of sternal infection. In the long term it can lead to chronic pain and significant deformity (Fig. 1). To prevent this complication we have designed and 


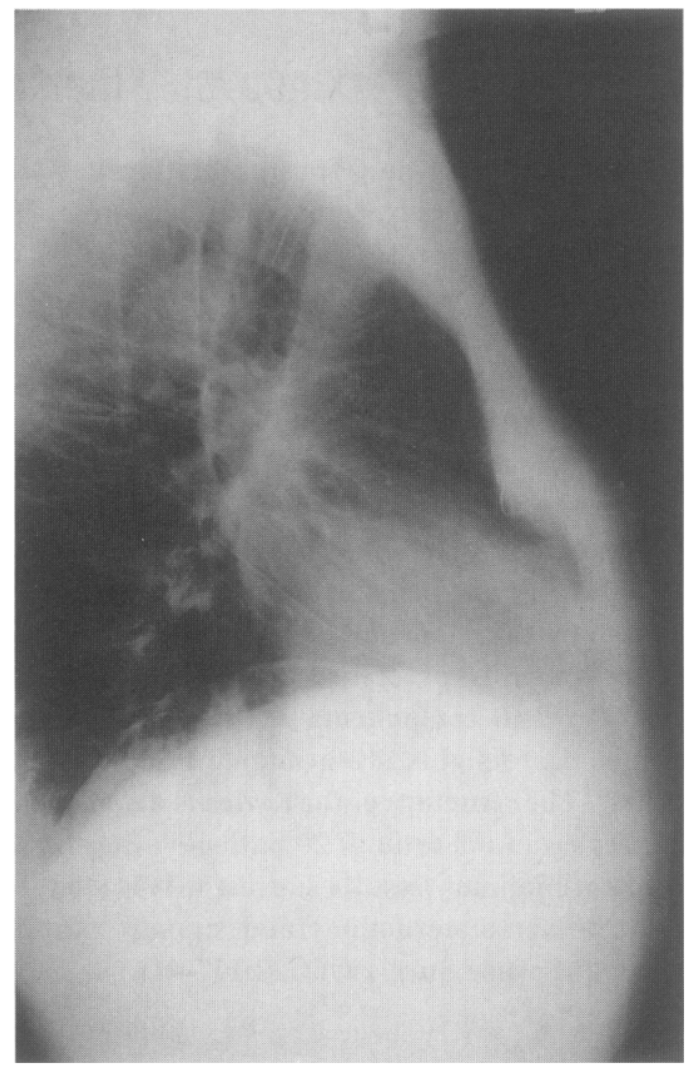

Fig. 1. Lateral chest radiograph demonstrating sternal disruption at transverse sternotomy site. Note anterior displacement of inferior sternal part and fractured sternal wires.

constructed a sternal fixation device. The device in situ is demonstrated in Fig. 2. The method associated with its design and provisional results are provided in this article.

\section{Method}

The underlying problem that needs to be overcome in this group of patients is the anterior displacement of the inferior sternal part at the transverse sternotomy site (Fig. 1). This is demonstrated clearly in all cases of sternal disruption after the procedure. The reason for this anterior displacement is unclear, but it may be a manifestation of the now-unopposed action of the rectus abdominus muscles in combination with postoperative alteration to breathing mechanics. In our experience these forces are considerable, and the sternal junction at the sternotomy site offers little resistance to displacement because of the small contact surface area.

Obstacles one must overcome when designing a device to fixate the sternum at this level include the anatomic structure of the sternum with its two thin outer cortices and the space limitations at this site. Device thickness is restricted anteriorly by scant overlying muscle and subcu-

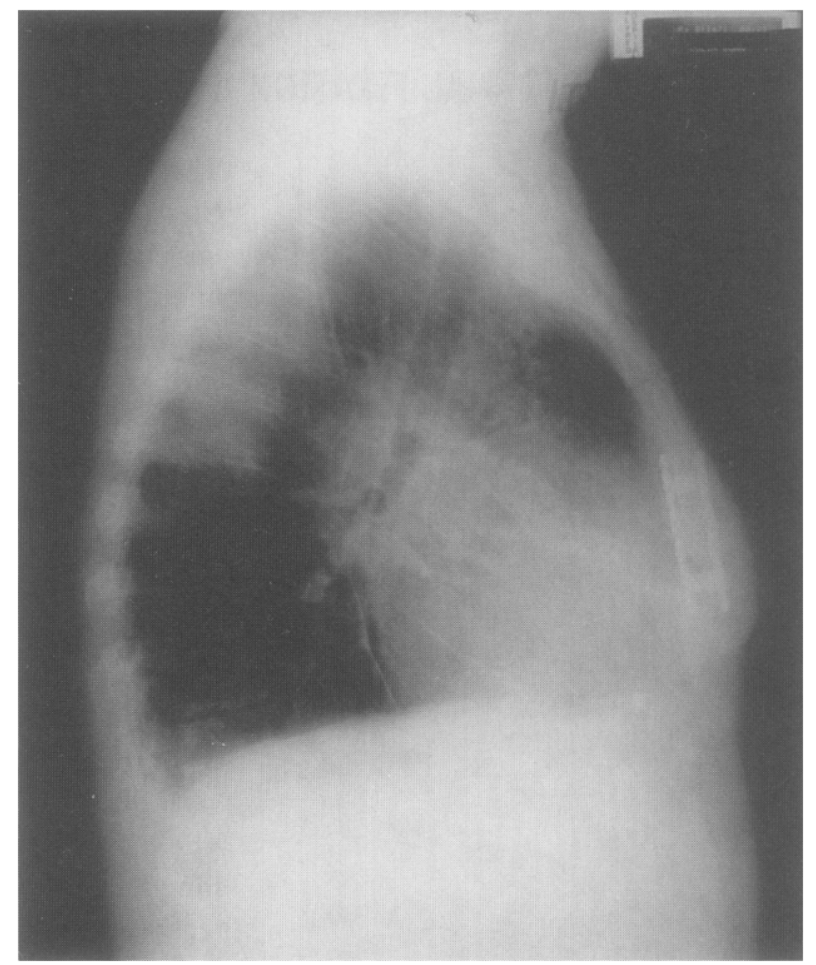

Fig. 2. Lateral chest radiograph demonstrating sternal fixation with device.

taneous tissue and posteriorly by the proximity of the mediastinum: device protrusion may cause skin erosion or mediastinal damage.

The relative fragility of the sternum does not provide much strength for fixation by conventional devices. The device we believe to be the ideal structure to fixate the sternum at this level consists of a two-plate system, one plate for each side of the sternum, in which the plates are fixed to each other by means of two posts (Fig. 3). This provides its own inherent strength and eliminates the inadequacy of the sternum itself. The strength of the plate material is such that only thin plates are required. The posts are fixed to the posterior plate to form a solitary unit, and this combined with countersinking of the screws that fix the anterior plate to the posts overcomes space restrictions in this area while also avoiding any prominence. The posts are sunk into the sternum via drilled holes a predetermined distance from the sternotomy edge. This provides a large mass of bone to resist displacing forces. An in situ illustration of the device both anteriorly and in cross section is shown in Fig. 4.

Device dimensions were obtained by modeling the device to resist the determined forces while complying with the space restrictions. With the device in situ, the force exerted by attempted anterior displacement of the sternum is translated into a distraction force exerted by the post on the bone between the post hole and the sternotomy edge. This is to a greater extent on the upper sternal part. This distraction force is distributed along the circumference of the post hole. ${ }^{2}$ 


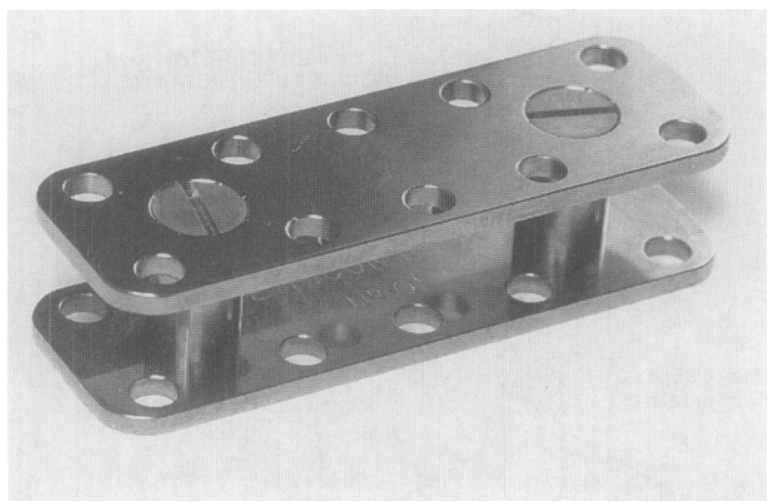

Fig. 3. Sternal fixation device.

The force exerted by the plates on the anterior and posterior surfaces of the sternum is not a significant factor as regards prevention of disruption.

The major factors that prevent bone disruption are therefore the diameter of the post and the distance between the post hole and transverse sternotomy, with a greater emphasis on the latter on the superior sternal part. Both these factors will be inversely proportional to the likelihood of disruption of the sternum. The most vulnerable aspect of the sternum is that which exists between the superior sternal post and the transverse sternotomy edge.

The dimensions of the device that we believe to be the most appropriate to prevent sternal disruption while complying with space restrictions are shown in Fig. 5.

Method of insertion of the device. The incision is made to the point at which only transverse division of the sternum is required for its completion. At this point, the sternum is marked with a diathermy instrument at the proposed sternotomy site. A small amount of tissue mobilization is required anteriorly to accommodate the device. Complete denudation of the sternum is not necessary because peristernal tissue enhances device fixation. The template (Fig. 6) is placed on the sternum with its site location line corresponding to the diathermy mark. The two holes are then drilled through the superior and inferior sternal parts at the designated points. This may be done at any stage but is easier done before the sternotomy. A protective guide is attached to the twist drill to prevent damage to the underlying mediastinum. An $8 \mathrm{~mm}$ drill bit is used for the post holes and the distance between these on the template makes allowance for the bony deficit that will result from the sternotomy. Note from the device dimensions (Fig. 5) that the distance from the post hole to the sternotomy site is different for each sternal part. Once the holes have been drilled, the sternal thickness is measured and the appropriate-size device is chosen. This is done with use of the guide on the handle aspect of the template (Fig. 6). In our experience it is preferable to go down one size $(1 \mathrm{~mm})$ on the measured thickness. This results in more solid fixation. The operation is then done in the standard fashion. 1,3

On completion of the operation and at the time for closure (after loose application of pericostal sutures if this is the desired technique), the post for the inferior sternal
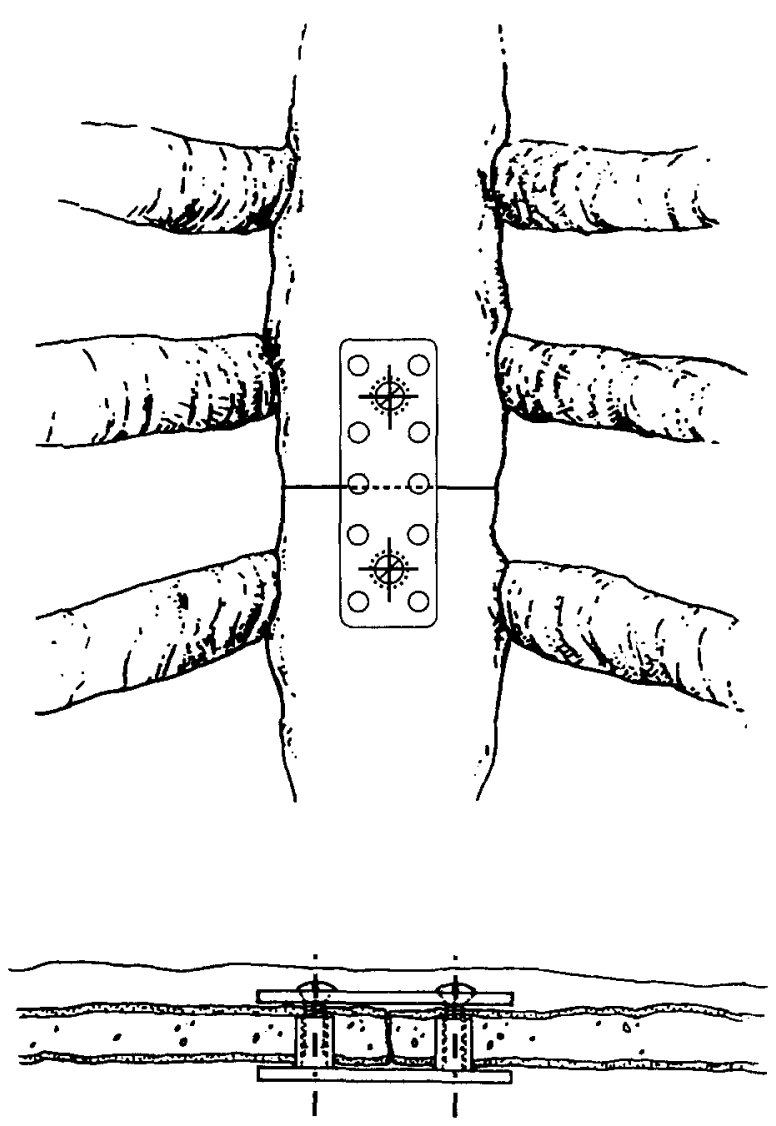

Fig. 4. Device in situ viewed from anterior aspect of sternum and in cross section.

part is inserted from the posterior aspect of the sternum through its designated hole. The top plate is screwed loosely into this post and rotated laterally 90 degrees. This holds the device in position. Two rib approximaters are then used, one on each side of the sternum, and these are tightened to lower the superior sternal part slowly down to its premorbid position. The superior sternal part post hole is positioned over its designated post. Once this is in position, the top plate is rotated back and its screw inserted. Both screws are then firmly tightened and fixation is complete. The remainder of the wound is then closed in the routine fashion.

\section{Results}

To date a total of 20 sternal fixation devices have been inserted at the primary procedure of bilateral sequential single-lung transplantation with use of the transsternal bilateral thoracotomy incision. There have been no sternal disruptions or significant sternal mobility in either the immediate or longterm postoperative period with follow-up now as much as 12 months. There was a minor degree of 

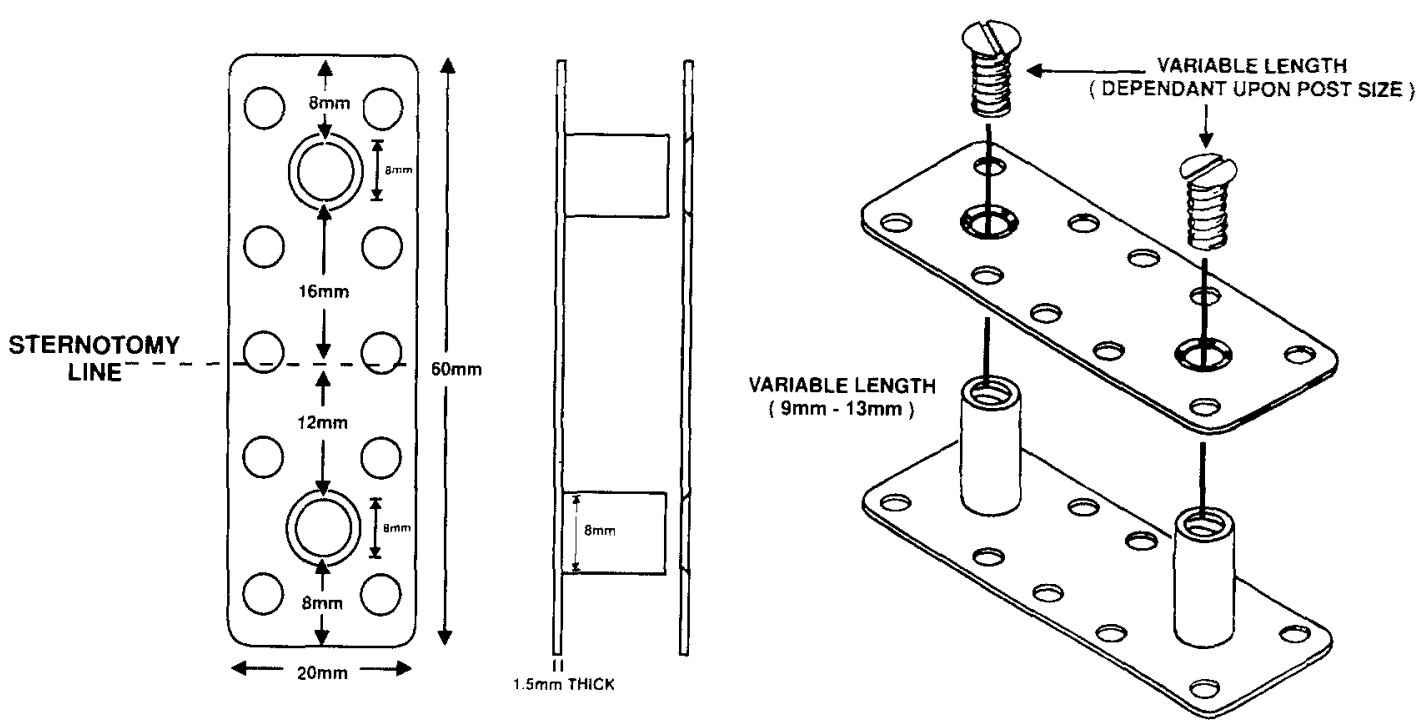

Fig. 5. Ideal device dimensions.

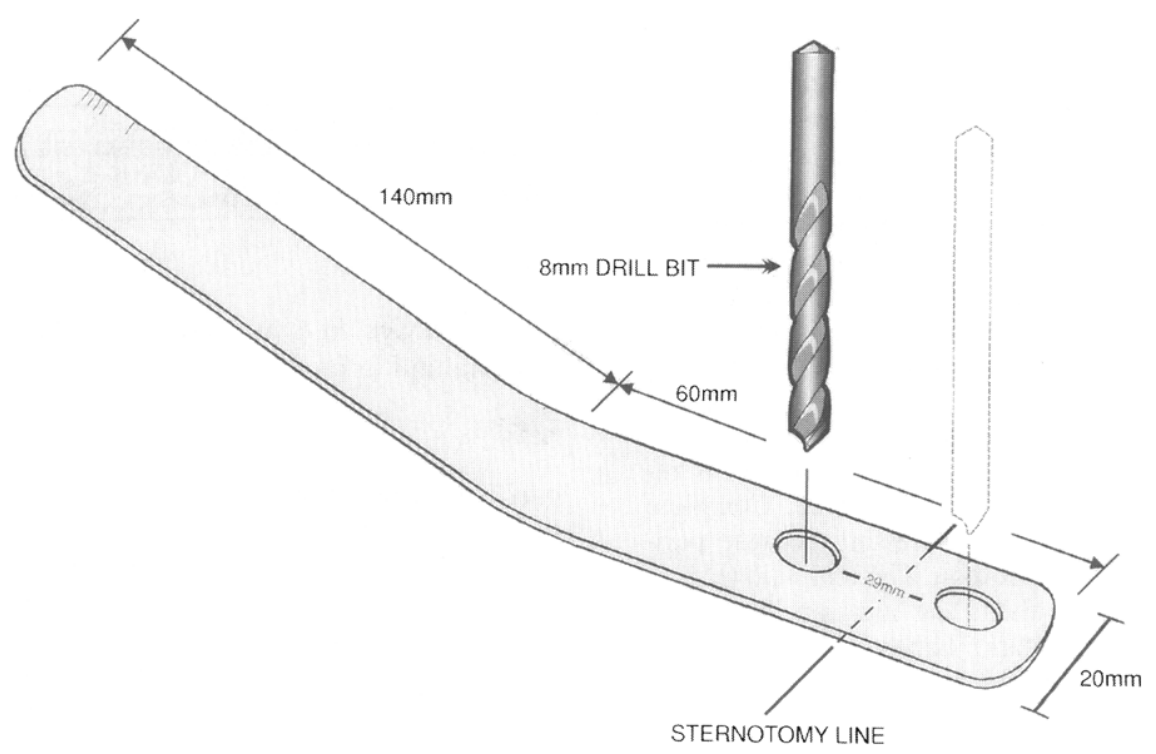

Fig. 6. Template required for determination of sternal thickness and selection of sternal hole sites.

mobility detected early in one patient, which resolved with no additional intervention. There have been no sternal infections. These results compare favorably with those in the 11 transplant procedures done before the introduction of the device, among which there were four sternal disruptions $(36 \%)$ and one sternal infection.

An area of concern with the use of such a device is the ease at which it can be removed at a later date if required. One patient required a median sternot- omy at 10 months after transplant because of an unrelated problem and at operation it was found that the sternum was well healed and the device was able to be removed before sternotomy.

\section{Discussion}

Bilateral sequential single-lung transplantation is the most rapidly growing transplant procedure. The prevention of disruption at the transverse sternotomy site of the transverse thoracosternotomy inci- 
sion will lead to significantly decreased morbidity in both the early and late postoperative periods. We believe we now have a device that significantly decreases the prevalence of sternal disruption and this is borne out by our preliminary results. We have now done 20 insertions with no complications, compared with a predevice disruption rate of $36 \%$. The follow-up period is now as great as 12 months. We advocate the routine use of this device in the closure of any transverse sternotomy. Further applications may develop with continued use of the device, and its use in fixation of sternal fractures is being investigated.

\section{REFERENCES}

1. Pasque MK, Cooper JD, Kaiser LR, et al. Improved technique for bilateral lung transplantation: rationale and clinical experience. Ann Thorac Surg 1990;49:785-79.

2. Gianncoli DC. Physics for scientists and engineers with modern physics. 2nd ed. Englewood Cliffs, New Jersey: PrenticeHall, 1984.

3. Esmore DS, Brown RP, Buckland MB, et al. Techniques and results in bilateral sequential single lung transplantation. J Card Surg 1994;9:1-14.

\section{Availability of Journal back issues}

As a service to our subscribers, copies of back issues of The Journal of Thoracic and Cardiovascular Surgery for the preceding 5 years are maintained and are available for purchase from Mosby until inventory is depleted at a cost of $\$ 13.50$ per issue. The following quantity discounts are available: $25 \%$ off on quantities of 12 to 23 , and one third off on quantities of 24 or more. Please write to Mosby-Year Book, Inc., Subscription Services, 11830 Westline Industrial Drive, St. Louis MO 63146-3318, or call 800-453-4351 or 314-453-4351 for information on availability of particular issues. If unavailable from the publisher, photocopies of complete issues may be purchased from UMI, 300 N. Zeeb Rd., Ann Arbor, MI 48106, 313-761-4700. 